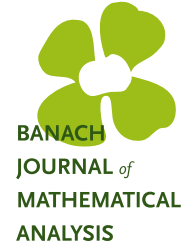

Banach J. Math. Anal. 10 (2016), no. 3, 482-494

http://dx.doi.org/10.1215/17358787-3599741

ISSN: $1735-8787$ (electronic)

http://projecteuclid.org/bjma

ANALYSIS

\title{
ON CERTAIN UNIFORMLY OPEN MULTILINEAR MAPPINGS
}

\author{
MAREK BALCERZAK, ${ }^{1}$ EHRHARD BEHRENDS, ${ }^{2}$ and FILIP STROBIN ${ }^{1 *}$ \\ Communicated by G. Popescu
}

\begin{abstract}
We obtain two results stating the uniform openness of bilinear operators and multilinear functionals. The first result deals with Banach spaces $L^{p}:=L_{\mathbb{K}}^{p}$ (over $\mathbb{K} \in\{\mathbb{R}, \mathbb{C}\}$ ) and pointwise multiplication from $L^{p} \times L^{q}$ to $L^{r}$ (where $1 / p+1 / q=1 / r$ ). The second result is concerned with the nontrivial $n$-linear functionals from the product $X_{1} \times \cdots \times X_{n}$ of normed spaces (over $\mathbb{K} \in\{\mathbb{R}, \mathbb{C}\})$ to the field $\mathbb{K}$.
\end{abstract}

\section{IntroduCtion}

Assume that $X$ and $Y$ are metric spaces. A mapping $T: X \rightarrow Y$ is called open if it sends every open set in $X$ to an open set in $Y$. For $x_{0} \in X$ we say that $T$ is (locally) open at $x_{0}$ if for every $\varepsilon>0$ there exists $\delta>0$ such that $B\left(T\left(x_{0}\right), \delta\right) \subset T\left[B\left(x_{0}, \varepsilon\right)\right]$ (see [8, Section 13, XIII]). Here $B(z, r)$ stands for the open ball with center $z$ and radius $r>0$ in a given space. Note that in the above definition one can use closed balls instead of open balls. Obviously, $T$ is open if and only if it is open at every $x \in X$. Note that the notion of local openness is still interesting for various mappings in topology (see, e.g., [5]).

In [1], $T$ is called uniformly open if $T$ is locally open at every $x$ and, for given $\varepsilon$, the $\delta$ can be chosen such that it does not depend on $x$. An open mapping need not be uniformly open; the function arctan serves as an easy example.

The classical Banach open mapping principle states that every linear continuous surjection between two Banach spaces is an open mapping. In fact, it is

Copyright 2016 by the Tusi Mathematical Research Group.

Received Jul. 13, 2015; Accepted Oct. 2, 2015.

${ }^{*}$ Corresponding author.

2010 Mathematics Subject Classification. Primary 46B25; Secondary 47A06, 47A07, 54C10.

Keywords. multiplication, uniformly open mapping, $L_{p}$ spaces, multilinear functionals. 


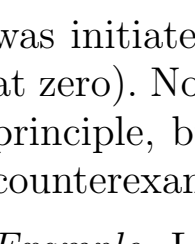

Banach J. Math. Anal. 10 (2016), no. 3, 482-494

http://dx.doi.org/10.1215/17358787-3599741

ISSN: $1735-8787$ (electronic)

http://projecteuclid.org/bjma

ANALYSIS

\title{
ON CERTAIN UNIFORMLY OPEN MULTILINEAR MAPPINGS
}

\author{
MAREK BALCERZAK, ${ }^{1}$ EHRHARD BEHRENDS, ${ }^{2}$ and FILIP STROBIN ${ }^{1 *}$ \\ Communicated by G. Popescu
}

\begin{abstract}
We obtain two results stating the uniform openness of bilinear operators and multilinear functionals. The first result deals with Banach spaces $L^{p}:=L_{\mathbb{K}}^{p}$ (over $\mathbb{K} \in\{\mathbb{R}, \mathbb{C}\}$ ) and pointwise multiplication from $L^{p} \times L^{q}$ to $L^{r}$ (where $1 / p+1 / q=1 / r$ ). The second result is concerned with the nontrivial $n$-linear functionals from the product $X_{1} \times \cdots \times X_{n}$ of normed spaces (over $\mathbb{K} \in\{\mathbb{R}, \mathbb{C}\})$ to the field $\mathbb{K}$.
\end{abstract}

\section{IntroduCtion}

Assume that $X$ and $Y$ are metric spaces. A mapping $T: X \rightarrow Y$ is called open if it sends every open set in $X$ to an open set in $Y$. For $x_{0} \in X$ we say that $T$ is (locally) open at $x_{0}$ if for every $\varepsilon>0$ there exists $\delta>0$ such that $B\left(T\left(x_{0}\right), \delta\right) \subset T\left[B\left(x_{0}, \varepsilon\right)\right]$ (see [8, Section 13, XIII]). Here $B(z, r)$ stands for the open ball with center $z$ and radius $r>0$ in a given space. Note that in the above definition one can use closed balls instead of open balls. Obviously, $T$ is open if and only if it is open at every $x \in X$. Note that the notion of local openness is still interesting for various mappings in topology (see, e.g., [5]).

In [1], $T$ is called uniformly open if $T$ is locally open at every $x$ and, for given $\varepsilon$, the $\delta$ can be chosen such that it does not depend on $x$. An open mapping need not be uniformly open; the function arctan serves as an easy example.

The classical Banach open mapping principle states that every linear continuous surjection between two Banach spaces is an open mapping. In fact, it is

Copyright 2016 by the Tusi Mathematical Research Group.

Received Jul. 13, 2015; Accepted Oct. 2, 2015.

${ }^{*}$ Corresponding author.

2010 Mathematics Subject Classification. Primary 46B25; Secondary 47A06, 47A07, 54C10.

Keywords. multiplication, uniformly open mapping, $L_{p}$ spaces, multilinear functionals. 


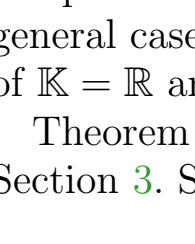

Banach J. Math. Anal. 10 (2016), no. 3, 482-494

http://dx.doi.org/10.1215/17358787-3599741

ISSN: $1735-8787$ (electronic)

http://projecteuclid.org/bjma

ANALYSIS

\title{
ON CERTAIN UNIFORMLY OPEN MULTILINEAR MAPPINGS
}

\author{
MAREK BALCERZAK, ${ }^{1}$ EHRHARD BEHRENDS, ${ }^{2}$ and FILIP STROBIN ${ }^{1 *}$ \\ Communicated by G. Popescu
}

\begin{abstract}
We obtain two results stating the uniform openness of bilinear operators and multilinear functionals. The first result deals with Banach spaces $L^{p}:=L_{\mathbb{K}}^{p}$ (over $\mathbb{K} \in\{\mathbb{R}, \mathbb{C}\}$ ) and pointwise multiplication from $L^{p} \times L^{q}$ to $L^{r}$ (where $1 / p+1 / q=1 / r$ ). The second result is concerned with the nontrivial $n$-linear functionals from the product $X_{1} \times \cdots \times X_{n}$ of normed spaces (over $\mathbb{K} \in\{\mathbb{R}, \mathbb{C}\})$ to the field $\mathbb{K}$.
\end{abstract}

\section{IntroduCtion}

Assume that $X$ and $Y$ are metric spaces. A mapping $T: X \rightarrow Y$ is called open if it sends every open set in $X$ to an open set in $Y$. For $x_{0} \in X$ we say that $T$ is (locally) open at $x_{0}$ if for every $\varepsilon>0$ there exists $\delta>0$ such that $B\left(T\left(x_{0}\right), \delta\right) \subset T\left[B\left(x_{0}, \varepsilon\right)\right]$ (see [8, Section 13, XIII]). Here $B(z, r)$ stands for the open ball with center $z$ and radius $r>0$ in a given space. Note that in the above definition one can use closed balls instead of open balls. Obviously, $T$ is open if and only if it is open at every $x \in X$. Note that the notion of local openness is still interesting for various mappings in topology (see, e.g., [5]).

In [1], $T$ is called uniformly open if $T$ is locally open at every $x$ and, for given $\varepsilon$, the $\delta$ can be chosen such that it does not depend on $x$. An open mapping need not be uniformly open; the function arctan serves as an easy example.

The classical Banach open mapping principle states that every linear continuous surjection between two Banach spaces is an open mapping. In fact, it is

Copyright 2016 by the Tusi Mathematical Research Group.

Received Jul. 13, 2015; Accepted Oct. 2, 2015.

${ }^{*}$ Corresponding author.

2010 Mathematics Subject Classification. Primary 46B25; Secondary 47A06, 47A07, 54C10.

Keywords. multiplication, uniformly open mapping, $L_{p}$ spaces, multilinear functionals. 


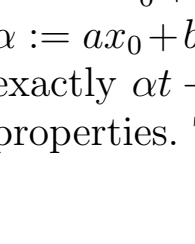

Banach J. Math. Anal. 10 (2016), no. 3, 482-494

http://dx.doi.org/10.1215/17358787-3599741

ISSN: $1735-8787$ (electronic)

http://projecteuclid.org/bjma

ANALYSIS

\title{
ON CERTAIN UNIFORMLY OPEN MULTILINEAR MAPPINGS
}

\author{
MAREK BALCERZAK, ${ }^{1}$ EHRHARD BEHRENDS, ${ }^{2}$ and FILIP STROBIN ${ }^{1 *}$ \\ Communicated by G. Popescu
}

\begin{abstract}
We obtain two results stating the uniform openness of bilinear operators and multilinear functionals. The first result deals with Banach spaces $L^{p}:=L_{\mathbb{K}}^{p}$ (over $\mathbb{K} \in\{\mathbb{R}, \mathbb{C}\}$ ) and pointwise multiplication from $L^{p} \times L^{q}$ to $L^{r}$ (where $1 / p+1 / q=1 / r$ ). The second result is concerned with the nontrivial $n$-linear functionals from the product $X_{1} \times \cdots \times X_{n}$ of normed spaces (over $\mathbb{K} \in\{\mathbb{R}, \mathbb{C}\})$ to the field $\mathbb{K}$.
\end{abstract}

\section{IntroduCtion}

Assume that $X$ and $Y$ are metric spaces. A mapping $T: X \rightarrow Y$ is called open if it sends every open set in $X$ to an open set in $Y$. For $x_{0} \in X$ we say that $T$ is (locally) open at $x_{0}$ if for every $\varepsilon>0$ there exists $\delta>0$ such that $B\left(T\left(x_{0}\right), \delta\right) \subset T\left[B\left(x_{0}, \varepsilon\right)\right]$ (see [8, Section 13, XIII]). Here $B(z, r)$ stands for the open ball with center $z$ and radius $r>0$ in a given space. Note that in the above definition one can use closed balls instead of open balls. Obviously, $T$ is open if and only if it is open at every $x \in X$. Note that the notion of local openness is still interesting for various mappings in topology (see, e.g., [5]).

In [1], $T$ is called uniformly open if $T$ is locally open at every $x$ and, for given $\varepsilon$, the $\delta$ can be chosen such that it does not depend on $x$. An open mapping need not be uniformly open; the function arctan serves as an easy example.

The classical Banach open mapping principle states that every linear continuous surjection between two Banach spaces is an open mapping. In fact, it is

Copyright 2016 by the Tusi Mathematical Research Group.

Received Jul. 13, 2015; Accepted Oct. 2, 2015.

${ }^{*}$ Corresponding author.

2010 Mathematics Subject Classification. Primary 46B25; Secondary 47A06, 47A07, 54C10.

Keywords. multiplication, uniformly open mapping, $L_{p}$ spaces, multilinear functionals. 


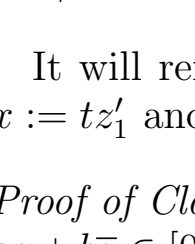

Banach J. Math. Anal. 10 (2016), no. 3, 482-494

http://dx.doi.org/10.1215/17358787-3599741

ISSN: $1735-8787$ (electronic)

http://projecteuclid.org/bjma

ANALYSIS

\title{
ON CERTAIN UNIFORMLY OPEN MULTILINEAR MAPPINGS
}

\author{
MAREK BALCERZAK, ${ }^{1}$ EHRHARD BEHRENDS, ${ }^{2}$ and FILIP STROBIN ${ }^{1 *}$ \\ Communicated by G. Popescu
}

\begin{abstract}
We obtain two results stating the uniform openness of bilinear operators and multilinear functionals. The first result deals with Banach spaces $L^{p}:=L_{\mathbb{K}}^{p}$ (over $\mathbb{K} \in\{\mathbb{R}, \mathbb{C}\}$ ) and pointwise multiplication from $L^{p} \times L^{q}$ to $L^{r}$ (where $1 / p+1 / q=1 / r$ ). The second result is concerned with the nontrivial $n$-linear functionals from the product $X_{1} \times \cdots \times X_{n}$ of normed spaces (over $\mathbb{K} \in\{\mathbb{R}, \mathbb{C}\})$ to the field $\mathbb{K}$.
\end{abstract}

\section{IntroduCtion}

Assume that $X$ and $Y$ are metric spaces. A mapping $T: X \rightarrow Y$ is called open if it sends every open set in $X$ to an open set in $Y$. For $x_{0} \in X$ we say that $T$ is (locally) open at $x_{0}$ if for every $\varepsilon>0$ there exists $\delta>0$ such that $B\left(T\left(x_{0}\right), \delta\right) \subset T\left[B\left(x_{0}, \varepsilon\right)\right]$ (see [8, Section 13, XIII]). Here $B(z, r)$ stands for the open ball with center $z$ and radius $r>0$ in a given space. Note that in the above definition one can use closed balls instead of open balls. Obviously, $T$ is open if and only if it is open at every $x \in X$. Note that the notion of local openness is still interesting for various mappings in topology (see, e.g., [5]).

In [1], $T$ is called uniformly open if $T$ is locally open at every $x$ and, for given $\varepsilon$, the $\delta$ can be chosen such that it does not depend on $x$. An open mapping need not be uniformly open; the function arctan serves as an easy example.

The classical Banach open mapping principle states that every linear continuous surjection between two Banach spaces is an open mapping. In fact, it is

Copyright 2016 by the Tusi Mathematical Research Group.

Received Jul. 13, 2015; Accepted Oct. 2, 2015.

${ }^{*}$ Corresponding author.

2010 Mathematics Subject Classification. Primary 46B25; Secondary 47A06, 47A07, 54C10.

Keywords. multiplication, uniformly open mapping, $L_{p}$ spaces, multilinear functionals. 


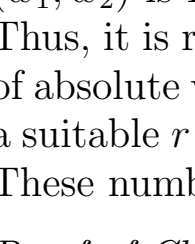

Banach J. Math. Anal. 10 (2016), no. 3, 482-494

http://dx.doi.org/10.1215/17358787-3599741

ISSN: $1735-8787$ (electronic)

http://projecteuclid.org/bjma

ANALYSIS

\title{
ON CERTAIN UNIFORMLY OPEN MULTILINEAR MAPPINGS
}

\author{
MAREK BALCERZAK, ${ }^{1}$ EHRHARD BEHRENDS, ${ }^{2}$ and FILIP STROBIN ${ }^{1 *}$ \\ Communicated by G. Popescu
}

\begin{abstract}
We obtain two results stating the uniform openness of bilinear operators and multilinear functionals. The first result deals with Banach spaces $L^{p}:=L_{\mathbb{K}}^{p}$ (over $\mathbb{K} \in\{\mathbb{R}, \mathbb{C}\}$ ) and pointwise multiplication from $L^{p} \times L^{q}$ to $L^{r}$ (where $1 / p+1 / q=1 / r$ ). The second result is concerned with the nontrivial $n$-linear functionals from the product $X_{1} \times \cdots \times X_{n}$ of normed spaces (over $\mathbb{K} \in\{\mathbb{R}, \mathbb{C}\})$ to the field $\mathbb{K}$.
\end{abstract}

\section{IntroduCtion}

Assume that $X$ and $Y$ are metric spaces. A mapping $T: X \rightarrow Y$ is called open if it sends every open set in $X$ to an open set in $Y$. For $x_{0} \in X$ we say that $T$ is (locally) open at $x_{0}$ if for every $\varepsilon>0$ there exists $\delta>0$ such that $B\left(T\left(x_{0}\right), \delta\right) \subset T\left[B\left(x_{0}, \varepsilon\right)\right]$ (see [8, Section 13, XIII]). Here $B(z, r)$ stands for the open ball with center $z$ and radius $r>0$ in a given space. Note that in the above definition one can use closed balls instead of open balls. Obviously, $T$ is open if and only if it is open at every $x \in X$. Note that the notion of local openness is still interesting for various mappings in topology (see, e.g., [5]).

In [1], $T$ is called uniformly open if $T$ is locally open at every $x$ and, for given $\varepsilon$, the $\delta$ can be chosen such that it does not depend on $x$. An open mapping need not be uniformly open; the function arctan serves as an easy example.

The classical Banach open mapping principle states that every linear continuous surjection between two Banach spaces is an open mapping. In fact, it is

Copyright 2016 by the Tusi Mathematical Research Group.

Received Jul. 13, 2015; Accepted Oct. 2, 2015.

${ }^{*}$ Corresponding author.

2010 Mathematics Subject Classification. Primary 46B25; Secondary 47A06, 47A07, 54C10.

Keywords. multiplication, uniformly open mapping, $L_{p}$ spaces, multilinear functionals. 


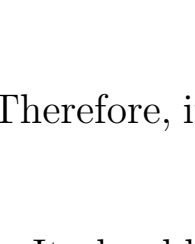

Banach J. Math. Anal. 10 (2016), no. 3, 482-494

http://dx.doi.org/10.1215/17358787-3599741

ISSN: $1735-8787$ (electronic)

http://projecteuclid.org/bjma

ANALYSIS

\title{
ON CERTAIN UNIFORMLY OPEN MULTILINEAR MAPPINGS
}

\author{
MAREK BALCERZAK, ${ }^{1}$ EHRHARD BEHRENDS, ${ }^{2}$ and FILIP STROBIN ${ }^{1 *}$ \\ Communicated by G. Popescu
}

\begin{abstract}
We obtain two results stating the uniform openness of bilinear operators and multilinear functionals. The first result deals with Banach spaces $L^{p}:=L_{\mathbb{K}}^{p}$ (over $\mathbb{K} \in\{\mathbb{R}, \mathbb{C}\}$ ) and pointwise multiplication from $L^{p} \times L^{q}$ to $L^{r}$ (where $1 / p+1 / q=1 / r$ ). The second result is concerned with the nontrivial $n$-linear functionals from the product $X_{1} \times \cdots \times X_{n}$ of normed spaces (over $\mathbb{K} \in\{\mathbb{R}, \mathbb{C}\})$ to the field $\mathbb{K}$.
\end{abstract}

\section{IntroduCtion}

Assume that $X$ and $Y$ are metric spaces. A mapping $T: X \rightarrow Y$ is called open if it sends every open set in $X$ to an open set in $Y$. For $x_{0} \in X$ we say that $T$ is (locally) open at $x_{0}$ if for every $\varepsilon>0$ there exists $\delta>0$ such that $B\left(T\left(x_{0}\right), \delta\right) \subset T\left[B\left(x_{0}, \varepsilon\right)\right]$ (see [8, Section 13, XIII]). Here $B(z, r)$ stands for the open ball with center $z$ and radius $r>0$ in a given space. Note that in the above definition one can use closed balls instead of open balls. Obviously, $T$ is open if and only if it is open at every $x \in X$. Note that the notion of local openness is still interesting for various mappings in topology (see, e.g., [5]).

In [1], $T$ is called uniformly open if $T$ is locally open at every $x$ and, for given $\varepsilon$, the $\delta$ can be chosen such that it does not depend on $x$. An open mapping need not be uniformly open; the function arctan serves as an easy example.

The classical Banach open mapping principle states that every linear continuous surjection between two Banach spaces is an open mapping. In fact, it is

Copyright 2016 by the Tusi Mathematical Research Group.

Received Jul. 13, 2015; Accepted Oct. 2, 2015.

${ }^{*}$ Corresponding author.

2010 Mathematics Subject Classification. Primary 46B25; Secondary 47A06, 47A07, 54C10.

Keywords. multiplication, uniformly open mapping, $L_{p}$ spaces, multilinear functionals. 


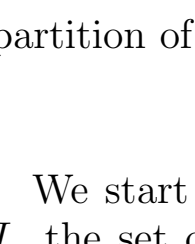

Banach J. Math. Anal. 10 (2016), no. 3, 482-494

http://dx.doi.org/10.1215/17358787-3599741

ISSN: $1735-8787$ (electronic)

http://projecteuclid.org/bjma

ANALYSIS

\title{
ON CERTAIN UNIFORMLY OPEN MULTILINEAR MAPPINGS
}

\author{
MAREK BALCERZAK, ${ }^{1}$ EHRHARD BEHRENDS, ${ }^{2}$ and FILIP STROBIN ${ }^{1 *}$ \\ Communicated by G. Popescu
}

\begin{abstract}
We obtain two results stating the uniform openness of bilinear operators and multilinear functionals. The first result deals with Banach spaces $L^{p}:=L_{\mathbb{K}}^{p}$ (over $\mathbb{K} \in\{\mathbb{R}, \mathbb{C}\}$ ) and pointwise multiplication from $L^{p} \times L^{q}$ to $L^{r}$ (where $1 / p+1 / q=1 / r$ ). The second result is concerned with the nontrivial $n$-linear functionals from the product $X_{1} \times \cdots \times X_{n}$ of normed spaces (over $\mathbb{K} \in\{\mathbb{R}, \mathbb{C}\})$ to the field $\mathbb{K}$.
\end{abstract}

\section{IntroduCtion}

Assume that $X$ and $Y$ are metric spaces. A mapping $T: X \rightarrow Y$ is called open if it sends every open set in $X$ to an open set in $Y$. For $x_{0} \in X$ we say that $T$ is (locally) open at $x_{0}$ if for every $\varepsilon>0$ there exists $\delta>0$ such that $B\left(T\left(x_{0}\right), \delta\right) \subset T\left[B\left(x_{0}, \varepsilon\right)\right]$ (see [8, Section 13, XIII]). Here $B(z, r)$ stands for the open ball with center $z$ and radius $r>0$ in a given space. Note that in the above definition one can use closed balls instead of open balls. Obviously, $T$ is open if and only if it is open at every $x \in X$. Note that the notion of local openness is still interesting for various mappings in topology (see, e.g., [5]).

In [1], $T$ is called uniformly open if $T$ is locally open at every $x$ and, for given $\varepsilon$, the $\delta$ can be chosen such that it does not depend on $x$. An open mapping need not be uniformly open; the function arctan serves as an easy example.

The classical Banach open mapping principle states that every linear continuous surjection between two Banach spaces is an open mapping. In fact, it is

Copyright 2016 by the Tusi Mathematical Research Group.

Received Jul. 13, 2015; Accepted Oct. 2, 2015.

${ }^{*}$ Corresponding author.

2010 Mathematics Subject Classification. Primary 46B25; Secondary 47A06, 47A07, 54C10.

Keywords. multiplication, uniformly open mapping, $L_{p}$ spaces, multilinear functionals. 


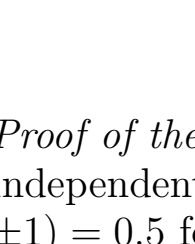

Banach J. Math. Anal. 10 (2016), no. 3, 482-494

http://dx.doi.org/10.1215/17358787-3599741

ISSN: $1735-8787$ (electronic)

http://projecteuclid.org/bjma

ANALYSIS

\title{
ON CERTAIN UNIFORMLY OPEN MULTILINEAR MAPPINGS
}

\author{
MAREK BALCERZAK, ${ }^{1}$ EHRHARD BEHRENDS, ${ }^{2}$ and FILIP STROBIN ${ }^{1 *}$ \\ Communicated by G. Popescu
}

\begin{abstract}
We obtain two results stating the uniform openness of bilinear operators and multilinear functionals. The first result deals with Banach spaces $L^{p}:=L_{\mathbb{K}}^{p}$ (over $\mathbb{K} \in\{\mathbb{R}, \mathbb{C}\}$ ) and pointwise multiplication from $L^{p} \times L^{q}$ to $L^{r}$ (where $1 / p+1 / q=1 / r$ ). The second result is concerned with the nontrivial $n$-linear functionals from the product $X_{1} \times \cdots \times X_{n}$ of normed spaces (over $\mathbb{K} \in\{\mathbb{R}, \mathbb{C}\})$ to the field $\mathbb{K}$.
\end{abstract}

\section{IntroduCtion}

Assume that $X$ and $Y$ are metric spaces. A mapping $T: X \rightarrow Y$ is called open if it sends every open set in $X$ to an open set in $Y$. For $x_{0} \in X$ we say that $T$ is (locally) open at $x_{0}$ if for every $\varepsilon>0$ there exists $\delta>0$ such that $B\left(T\left(x_{0}\right), \delta\right) \subset T\left[B\left(x_{0}, \varepsilon\right)\right]$ (see [8, Section 13, XIII]). Here $B(z, r)$ stands for the open ball with center $z$ and radius $r>0$ in a given space. Note that in the above definition one can use closed balls instead of open balls. Obviously, $T$ is open if and only if it is open at every $x \in X$. Note that the notion of local openness is still interesting for various mappings in topology (see, e.g., [5]).

In [1], $T$ is called uniformly open if $T$ is locally open at every $x$ and, for given $\varepsilon$, the $\delta$ can be chosen such that it does not depend on $x$. An open mapping need not be uniformly open; the function arctan serves as an easy example.

The classical Banach open mapping principle states that every linear continuous surjection between two Banach spaces is an open mapping. In fact, it is

Copyright 2016 by the Tusi Mathematical Research Group.

Received Jul. 13, 2015; Accepted Oct. 2, 2015.

${ }^{*}$ Corresponding author.

2010 Mathematics Subject Classification. Primary 46B25; Secondary 47A06, 47A07, 54C10.

Keywords. multiplication, uniformly open mapping, $L_{p}$ spaces, multilinear functionals. 


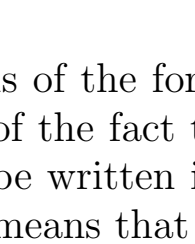

Banach J. Math. Anal. 10 (2016), no. 3, 482-494

http://dx.doi.org/10.1215/17358787-3599741

ISSN: $1735-8787$ (electronic)

http://projecteuclid.org/bjma

ANALYSIS

\title{
ON CERTAIN UNIFORMLY OPEN MULTILINEAR MAPPINGS
}

\author{
MAREK BALCERZAK, ${ }^{1}$ EHRHARD BEHRENDS, ${ }^{2}$ and FILIP STROBIN ${ }^{1 *}$ \\ Communicated by G. Popescu
}

\begin{abstract}
We obtain two results stating the uniform openness of bilinear operators and multilinear functionals. The first result deals with Banach spaces $L^{p}:=L_{\mathbb{K}}^{p}$ (over $\mathbb{K} \in\{\mathbb{R}, \mathbb{C}\}$ ) and pointwise multiplication from $L^{p} \times L^{q}$ to $L^{r}$ (where $1 / p+1 / q=1 / r$ ). The second result is concerned with the nontrivial $n$-linear functionals from the product $X_{1} \times \cdots \times X_{n}$ of normed spaces (over $\mathbb{K} \in\{\mathbb{R}, \mathbb{C}\})$ to the field $\mathbb{K}$.
\end{abstract}

\section{IntroduCtion}

Assume that $X$ and $Y$ are metric spaces. A mapping $T: X \rightarrow Y$ is called open if it sends every open set in $X$ to an open set in $Y$. For $x_{0} \in X$ we say that $T$ is (locally) open at $x_{0}$ if for every $\varepsilon>0$ there exists $\delta>0$ such that $B\left(T\left(x_{0}\right), \delta\right) \subset T\left[B\left(x_{0}, \varepsilon\right)\right]$ (see [8, Section 13, XIII]). Here $B(z, r)$ stands for the open ball with center $z$ and radius $r>0$ in a given space. Note that in the above definition one can use closed balls instead of open balls. Obviously, $T$ is open if and only if it is open at every $x \in X$. Note that the notion of local openness is still interesting for various mappings in topology (see, e.g., [5]).

In [1], $T$ is called uniformly open if $T$ is locally open at every $x$ and, for given $\varepsilon$, the $\delta$ can be chosen such that it does not depend on $x$. An open mapping need not be uniformly open; the function arctan serves as an easy example.

The classical Banach open mapping principle states that every linear continuous surjection between two Banach spaces is an open mapping. In fact, it is

Copyright 2016 by the Tusi Mathematical Research Group.

Received Jul. 13, 2015; Accepted Oct. 2, 2015.

${ }^{*}$ Corresponding author.

2010 Mathematics Subject Classification. Primary 46B25; Secondary 47A06, 47A07, 54C10.

Keywords. multiplication, uniformly open mapping, $L_{p}$ spaces, multilinear functionals. 


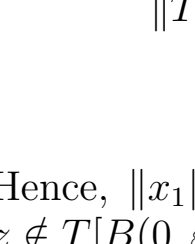

Banach J. Math. Anal. 10 (2016), no. 3, 482-494

http://dx.doi.org/10.1215/17358787-3599741

ISSN: $1735-8787$ (electronic)

http://projecteuclid.org/bjma

ANALYSIS

\title{
ON CERTAIN UNIFORMLY OPEN MULTILINEAR MAPPINGS
}

\author{
MAREK BALCERZAK, ${ }^{1}$ EHRHARD BEHRENDS, ${ }^{2}$ and FILIP STROBIN ${ }^{1 *}$ \\ Communicated by G. Popescu
}

\begin{abstract}
We obtain two results stating the uniform openness of bilinear operators and multilinear functionals. The first result deals with Banach spaces $L^{p}:=L_{\mathbb{K}}^{p}$ (over $\mathbb{K} \in\{\mathbb{R}, \mathbb{C}\}$ ) and pointwise multiplication from $L^{p} \times L^{q}$ to $L^{r}$ (where $1 / p+1 / q=1 / r$ ). The second result is concerned with the nontrivial $n$-linear functionals from the product $X_{1} \times \cdots \times X_{n}$ of normed spaces (over $\mathbb{K} \in\{\mathbb{R}, \mathbb{C}\})$ to the field $\mathbb{K}$.
\end{abstract}

\section{IntroduCtion}

Assume that $X$ and $Y$ are metric spaces. A mapping $T: X \rightarrow Y$ is called open if it sends every open set in $X$ to an open set in $Y$. For $x_{0} \in X$ we say that $T$ is (locally) open at $x_{0}$ if for every $\varepsilon>0$ there exists $\delta>0$ such that $B\left(T\left(x_{0}\right), \delta\right) \subset T\left[B\left(x_{0}, \varepsilon\right)\right]$ (see [8, Section 13, XIII]). Here $B(z, r)$ stands for the open ball with center $z$ and radius $r>0$ in a given space. Note that in the above definition one can use closed balls instead of open balls. Obviously, $T$ is open if and only if it is open at every $x \in X$. Note that the notion of local openness is still interesting for various mappings in topology (see, e.g., [5]).

In [1], $T$ is called uniformly open if $T$ is locally open at every $x$ and, for given $\varepsilon$, the $\delta$ can be chosen such that it does not depend on $x$. An open mapping need not be uniformly open; the function arctan serves as an easy example.

The classical Banach open mapping principle states that every linear continuous surjection between two Banach spaces is an open mapping. In fact, it is

Copyright 2016 by the Tusi Mathematical Research Group.

Received Jul. 13, 2015; Accepted Oct. 2, 2015.

${ }^{*}$ Corresponding author.

2010 Mathematics Subject Classification. Primary 46B25; Secondary 47A06, 47A07, 54C10.

Keywords. multiplication, uniformly open mapping, $L_{p}$ spaces, multilinear functionals. 


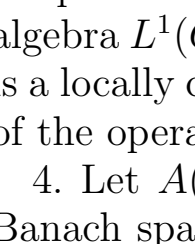

Banach J. Math. Anal. 10 (2016), no. 3, 482-494

http://dx.doi.org/10.1215/17358787-3599741

ISSN: $1735-8787$ (electronic)

http://projecteuclid.org/bjma

ANALYSIS

\title{
ON CERTAIN UNIFORMLY OPEN MULTILINEAR MAPPINGS
}

\author{
MAREK BALCERZAK, ${ }^{1}$ EHRHARD BEHRENDS, ${ }^{2}$ and FILIP STROBIN ${ }^{1 *}$ \\ Communicated by G. Popescu
}

\begin{abstract}
We obtain two results stating the uniform openness of bilinear operators and multilinear functionals. The first result deals with Banach spaces $L^{p}:=L_{\mathbb{K}}^{p}$ (over $\mathbb{K} \in\{\mathbb{R}, \mathbb{C}\}$ ) and pointwise multiplication from $L^{p} \times L^{q}$ to $L^{r}$ (where $1 / p+1 / q=1 / r$ ). The second result is concerned with the nontrivial $n$-linear functionals from the product $X_{1} \times \cdots \times X_{n}$ of normed spaces (over $\mathbb{K} \in\{\mathbb{R}, \mathbb{C}\})$ to the field $\mathbb{K}$.
\end{abstract}

\section{IntroduCtion}

Assume that $X$ and $Y$ are metric spaces. A mapping $T: X \rightarrow Y$ is called open if it sends every open set in $X$ to an open set in $Y$. For $x_{0} \in X$ we say that $T$ is (locally) open at $x_{0}$ if for every $\varepsilon>0$ there exists $\delta>0$ such that $B\left(T\left(x_{0}\right), \delta\right) \subset T\left[B\left(x_{0}, \varepsilon\right)\right]$ (see [8, Section 13, XIII]). Here $B(z, r)$ stands for the open ball with center $z$ and radius $r>0$ in a given space. Note that in the above definition one can use closed balls instead of open balls. Obviously, $T$ is open if and only if it is open at every $x \in X$. Note that the notion of local openness is still interesting for various mappings in topology (see, e.g., [5]).

In [1], $T$ is called uniformly open if $T$ is locally open at every $x$ and, for given $\varepsilon$, the $\delta$ can be chosen such that it does not depend on $x$. An open mapping need not be uniformly open; the function arctan serves as an easy example.

The classical Banach open mapping principle states that every linear continuous surjection between two Banach spaces is an open mapping. In fact, it is

Copyright 2016 by the Tusi Mathematical Research Group.

Received Jul. 13, 2015; Accepted Oct. 2, 2015.

${ }^{*}$ Corresponding author.

2010 Mathematics Subject Classification. Primary 46B25; Secondary 47A06, 47A07, 54C10.

Keywords. multiplication, uniformly open mapping, $L_{p}$ spaces, multilinear functionals. 


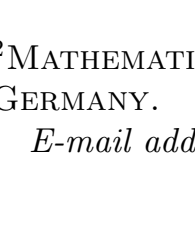

Banach J. Math. Anal. 10 (2016), no. 3, 482-494

http://dx.doi.org/10.1215/17358787-3599741

ISSN: $1735-8787$ (electronic)

http://projecteuclid.org/bjma

ANALYSIS

\title{
ON CERTAIN UNIFORMLY OPEN MULTILINEAR MAPPINGS
}

\author{
MAREK BALCERZAK, ${ }^{1}$ EHRHARD BEHRENDS, ${ }^{2}$ and FILIP STROBIN ${ }^{1 *}$ \\ Communicated by G. Popescu
}

\begin{abstract}
We obtain two results stating the uniform openness of bilinear operators and multilinear functionals. The first result deals with Banach spaces $L^{p}:=L_{\mathbb{K}}^{p}$ (over $\mathbb{K} \in\{\mathbb{R}, \mathbb{C}\}$ ) and pointwise multiplication from $L^{p} \times L^{q}$ to $L^{r}$ (where $1 / p+1 / q=1 / r$ ). The second result is concerned with the nontrivial $n$-linear functionals from the product $X_{1} \times \cdots \times X_{n}$ of normed spaces (over $\mathbb{K} \in\{\mathbb{R}, \mathbb{C}\})$ to the field $\mathbb{K}$.
\end{abstract}

\section{IntroduCtion}

Assume that $X$ and $Y$ are metric spaces. A mapping $T: X \rightarrow Y$ is called open if it sends every open set in $X$ to an open set in $Y$. For $x_{0} \in X$ we say that $T$ is (locally) open at $x_{0}$ if for every $\varepsilon>0$ there exists $\delta>0$ such that $B\left(T\left(x_{0}\right), \delta\right) \subset T\left[B\left(x_{0}, \varepsilon\right)\right]$ (see [8, Section 13, XIII]). Here $B(z, r)$ stands for the open ball with center $z$ and radius $r>0$ in a given space. Note that in the above definition one can use closed balls instead of open balls. Obviously, $T$ is open if and only if it is open at every $x \in X$. Note that the notion of local openness is still interesting for various mappings in topology (see, e.g., [5]).

In [1], $T$ is called uniformly open if $T$ is locally open at every $x$ and, for given $\varepsilon$, the $\delta$ can be chosen such that it does not depend on $x$. An open mapping need not be uniformly open; the function arctan serves as an easy example.

The classical Banach open mapping principle states that every linear continuous surjection between two Banach spaces is an open mapping. In fact, it is

Copyright 2016 by the Tusi Mathematical Research Group.

Received Jul. 13, 2015; Accepted Oct. 2, 2015.

${ }^{*}$ Corresponding author.

2010 Mathematics Subject Classification. Primary 46B25; Secondary 47A06, 47A07, 54C10.

Keywords. multiplication, uniformly open mapping, $L_{p}$ spaces, multilinear functionals. 